ВЕГЕТАТИВНОЕ ОБЕСПЕЧЕНИЕ ЦЕЛЕНАПРАВЛЕННОЙ ДЕЯТЕЛЬНОСТИ И ЕЁ РЕЗУЛЬТАТИВНОСТЬ У ПРАКТИЧЕСКИ ЗДОРОВЫХ ЛИЦ

\author{
(C) Р.А. Зорин, Ю.И. Медведева, И.С. Курепина, М.М. Лапкин, В.А. Жаднов
}

Рязанский государственный медицинский университет имени академика И.П. Павлова, Рязань, Российская Федерация

Цель. Изучение роли механизмов вегетативного обеспечения деятельности у практически здоровых людей в системогенезе её неодинаковой результативности.

Материалы и методы. Обследован 61 практически здоровый человек. Моделирование деятельности осуществлялось при помощи теста Шульте: выбор чисел разного цвета в порядке возрастания. До выполнения теста проводилось исследование вариабельности сердечного ритма с использованием методов анализа динамического ряда кардиоинтервалов и спектрального анализа и вызванных кожных симпатических потенциалов для оценки симпатических, парасимпатических, эрготропных и трофотропных регуляторных влияний. Выполнялся сравнительный анализ показателей методами непараметрической статистики, логит регрессионный анализ, моделирование зависимостей при помощи искусственных нейронных сетей.

Результаты. Была выявлена неоднородность группы исследуемых по показателям результативности деятельности: результативная группа характеризовалась меньшим числом ошибок, временем реакции после и до ошибки, низко результативная группа - противоположными характеристиками. При изучении механизмов вегетативного обеспечения целенаправленной деятельности человека выявлена большая активация эрготропных и трофотропных механизмов у испытуемых высоко результативной группы по показателям вызванных кожных симпатических потенциалов. Созданы модели логит-регрессионного анализа и искусственных нейронных сетей, обеспечивающие прогнозирование результативности деятельности на основе показателей вегетативного обеспечения. Наибольшее значение в решении задачи прогнозирования имели показатели вариабельности динамического ряда кардиоинтервалов. Модели, основанные на искусственных нейронных сетях, продемонстрировали более высокие классификационные показатели.

Заключение. Установлена роль влияния сегментарных и надсегментарных механизмов вегетативной регуляции в системной организации высокой результативности деятельности. Высокая результативность деятельности ассоциирована с большей активацией супрасегментарных эрготропных и трофотропных механизмов.

Ключевые слова: вегетативное обеспечение системной организации иеленаправленной деятельности человека, логит регрессионный анализ, искусственные нейронные сети.

\title{
THE VEGETATIVE SUPPORT OF PURPOSEFUL ACTIVITY AND PERFOMANCE EFFICIENCY IN HEALTHY PEOPLE
}

\author{
R.A. Zorin, Yu.I. Medvedeva, I.S. Kurepina, M.M. Lapkin, V.A. Zhadnov
}

Ryazan State Medical University, Ryazan, Russian Federation

Aim. The study of purposeful activity's autonomic maintenance and it's role in systemogenesis of unequal activity's efficiency in healthy people. 
Materials and Methods. 61 healthy persons were observed. The modeling of purposeful activity include Shulte test: the choice of numbers of different colors in ascending order. We recorded heart rate variability parameters and sympathetic skin evoked potentials before the Shulte test. The parameters of heart rate variability include analysis of cardio interval's dynamic range and spectral analysis characteristics. The heart rate variability parameters and sympathetic skin evoked potentials characteristics allowed us to evaluate sympathetic, parasympathetic, ergotropic and trophotropic regulative influences. Comparative analysis of characteristics by nonparametric methods, logit regression analysis, artificial neural networks modeling were performed.

Results. We identify the heterogeneity of investigated group by the parameters of activity's efficacy. The high performance group is characterized by lower level of mistakes, intervals after and before mistake; low performance group has opposite characteristics. A study of vegetative maintenance of modeling behavior revealed higher activation of ergotropic and trophotropic mechanisms in high performance group of healthy people. We offer some prognostic models which predicts the performance efficiency on the basis of vegetative maintenance parameters. The parameters of cardiointerval's variability play the most important role in solution of classification task. The artificial neural networks models have higher classification abilities.

Conclusion. The role of summary segmental and suprasegmental autonomic regulation influences in determination of system organization of high activity's efficiency was established. The high activity's performance is associated with activation of the suprasegmental ergotropic and trophotropic mechanisms.

Keywords: vegetative maintenance of human activity system organization activity, logit regression analysis, artificial neural networks.

Механизмы вегетативного обеспечения деятельности играют важную роль в определении её результативности, обеспечивая мобилизацию физиологических ресурсов и их восстановление, при этом особое значение имеет активация неспецифических стресс-реализующих и стресслимитирующих систем $[1,2]$.

Одними из основных принципов организации механизмов вегетативной регуляции являются принципы иерархических и реципрокных (функционально антагонистических) взаимоотношений различных уровней в рамках системной организации целенаправленного поведения человека [3]. Исследование вариабельности сердечного ритма как хорошо разработанный метод исследования автономной (вегетативной) нервной системы, тем не менее, не позволяет однозначно оценить сегментарные и супрасегментарные вегетативные влияния [4]. В связи с этим для изучения вегетативного обеспечения деятельности целесообразно использовать и другие методы исследования, в частности регистрацию и анализ вызванных кожных симпати- ческих потенциалов [5]. В целом такой подход может позволить установить роль сегментарных и надсегментарных механизмов вегетативной регуляции в системной организации целенаправленного поведения человека, нацеленной на достижение конкретного результата его деятельности.

Целью исследования является изучение роли механизмов вегетативного обеспечения в системной организации целенаправленного поведения у практически здоровых людей с неодинаковой результативностью моделируемой когнитивной деятельностью.

\section{Материалы и методы}

Обследовано 61 практически здоровый человек, из них 29 мужчин и 32 женщины. Возраст исследуемых $32,7 \pm 0,35$ лет. Критериями включения являлись возраст старше 18 лет, возможность выполнения условий исследования после предварительного инструктирования, ознакомление и подписание договора информированного согласия. Критериями исключения являлись наличие беременности, заболевания и травмы нервной системы, использование 
любых нейротропных препаратов в течение 1 года до начала исследования.

Исследование одобрено локальным этическим комитетом ФГБОУ ВО РязГМУ Минздрава России (2014), всеми пациентами до начала исследования подписан договор информированного согласия.

Целенаправленная деятельность реализовывалась при помощи теста Шульте: выбор чисел разного цвета на экране монитора в порядке возрастания при помощи комплекса для психофизиологического тестирования «НС-Психотест» (ООО «Нейрософт», Россия).

Оценка вегетативного обеспечения деятельности осуществлялась путём исследования вариабельности сердечного ритма (ВСР) и регистрации вызванных кожных симпатических потенциалов (ВКСП).

Для регистрации ВСР использовался прибор Варикард 2.5 (фирма «Рамена», Рязань) и программа «ИСКИМ» (версия 6.0). Оценивалась частота сердечных сокращений (ЧСС), среднее квадратичное отклонение (CKO); коэффициент вариации (CV), амплитуда моды (AМо), индекс напряжения (ИН) регуляторных систем (стрессиндекс - SI). Выполнен анализ мощности спектральных составляющих динамического ряда кардиоинтервалов с определением мощности дыхательных волн (HF); медленных волн 1 порядка (LF); медленных волн 2 порядка (VLF), суммарной мощности во всех диапазонах (Total Power, TP). Вычислялись показатели индекса централизации (ИЦ) как отношение HF+LF к VLF.

Регистрация ВКСП выполнена при помощи комплекса «Нейро-МВП» фирмы «Нейрософт» (Иваново). Проводилась запись ВКСП в положении исследуемого сидя с открытыми глазами до начала интеллектуальной деятельности. Регистрирующие электроды накладывались на кисти обеих рук, стимулирующий на указательный палец правой руки. Величина стимуляции составила в среднем 12 мА, длительность стимула 0,2 мс. Оценивались амплитудно-временные характеристики ВКСП: латентный период (ЛП), амплитуда первого (A1 - ассоциирован с трофотропными вегетативными влияними) и второго (А2 - ас- социирован с эрготропными влияниями) компонентов, длительность компонентов.

Обработка данных реализована с использованием программы Statistica $10.0 \mathrm{Ru}$. Применялся метод кластерного анализа [6]. Сравнительный анализ проводился с использованием непараметрического критерия Манна-Уитни (U), различия считались достоверными при уровне $\mathrm{p}<0,05$. Описательная характеристика подгрупп пациентов предполагала использование медианы, верхнего и нижнего квартиля (в формате [медиана, нижний квартиль, верхний квартиль]).

При применении логистического регрессионного анализа, уравнение регрессионного анализа представлено в стандартном виде $\mathrm{y}=\mathrm{b} 0+\mathrm{b} 1 * \mathrm{X} 1+\mathrm{b} 2 * \mathrm{X} 2 . . .+\mathrm{bn} * \mathrm{Xn}$; где $\mathrm{Y}$ - зависимая переменная, b0 - константа, b1, b2 ... bn - коэффициенты регрессии для независимых переменных, X1, $\mathrm{X} 2$... Xn - независимые переменные. Рассчитывалось значение вероятности распределения пациентов в подгруппы как отношение основания натурального логарифма в степени у к сумме натурального логарифма в степени у и 1. При значении вероятности распределения $<0,5$ исследуемый относился в группу 2, при значении больше 0,5 осуществлялось распределение в группу 1). В качестве функции потерь использовался метод максимального правдоподобия, в качестве критерия согласия использовалась статистика хи-квадрат и достигнутый руровень; оценивались отношения шансов для независимых переменных для определения их значимости в данной модели.

Моделирование искусственных нейронных сетей (ИНС) проводилось при помощи пакета программ Statistica $10.0(\mathrm{Ru})$. Наблюдения разделялись на обучающие, контрольные, тестовые выборки. Описывались выходные переменные (номер выделенных подгрупп испытуемых) и входные переменные (физиологические характеристики) $[7,8]$.

\section{Результаты и их обсуждение}

Методом кластерного анализа на основе выполнения теста Шульте выделено 2 группы исследуемых. В таблице 1 приведены характеристики выполнения теста Шульте в группах исследуемых. 


\section{Результаты теста Шульте в кластерах исследуемых}

\begin{tabular}{|c|c|c|c|c|}
\hline \multirow[b]{2}{*}{ Показатели } & Группа 1 & Группа 2 & \multirow[b]{2}{*}{$\mathrm{U}$} & \multirow[b]{2}{*}{$\mathrm{p}$} \\
\hline & $\begin{array}{c}\text { Медиана; нижний квартиль; } \\
\text { верхний квартиль }\end{array}$ & $\begin{array}{c}\text { [Медиана; нижний квартиль; } \\
\text { верхний квартиль] }\end{array}$ & & \\
\hline Интервал выбора, с & 2,$2 ; 1,5 ; 2,7$ & 1,$4 ; 0,9 ; 2,6$ & 21 & 0,070 \\
\hline Интервал после ошибки, с & 4,$7 ; 2,9 ; 6,5$ & 0,$5 ; 0,4 ; 0,8$ & 6,5 & 0,003 \\
\hline Интервал до ошибки, с & 1,$5 ; 0,9 ; 2,1$ & 0,$3 ; 0,2 ; 0,4$ & 6,3 & 0,003 \\
\hline Число ошибок & 4,$0 ; 1,0 ; 5,0$ & 0,$5 ; 0,2 ; 0,3$ & 7,5 & 0,001 \\
\hline
\end{tabular}

Как следует из таблицы в группе 1 (28 человек) определяется достоверно большее число ошибок, а также большее время выбора в связи ошибочным ответом по сравнению с группой 2 (33 человека).
При анализе показателей ВСР в выделенных группах достоверных различий не выявлено (табл. 2).

Вместе с тем, выявлены достоверные различия по характеристикам ВКСП в группах (табл. 3).

Таблица 2

\section{Показатели ВСР в группах исследуемых}

\begin{tabular}{|l|c|c|c|c|}
\hline \multirow{2}{*}{ Показатели } & Группа 1 & Группа 2 & \multirow{2}{*}{$\mathrm{v}$} \\
\cline { 2 - 3 } & $\begin{array}{c}\text { Медиана; нижний квартиль; } \\
\text { верхний квартиль }\end{array}$ & $\begin{array}{c}\text { Медиана; нижний квартиль; } \\
\text { верхний квартиль }\end{array}$ & \\
\hline ЧСС, в мин. & $77 ; 72 ; 88$ & $80 ; 73 ; 89$ & 203,5 & 0,549 \\
\hline CКО, мс & $44 ; 36 ; 62$ & $44 ; 30 ; 62$ & 227,5 & 0,990 \\
\hline AM0, \% & $39 ; 35 ; 42$ & $39 ; 36 ; 44$ & 208,5 & 0,633 \\
\hline ИН, усл. ед. & $154 ; 67 ; 204$ & $185 ; 86 ; 402$ & 187,5 & 0,322 \\
\hline HF, мc ${ }^{2}$ & $382 ; 209 ; 989$ & $343 ; 149 ; 1127$ & 211,5 & 0,687 \\
\hline LF, мc ${ }^{2}$ & $438 ; 248 ; 1130$ & $516 ; 277 ; 776$ & 223,5 & 0,912 \\
\hline VLF, мc $^{2}$ & $321 ; 182 ; 573$ & $180 ; 144 ; 415$ & 177,5 & 0,217 \\
\hline TP, мс ${ }^{2}$ & $1598 ; 740 ; 3360$ & $1175 ; 649 ; 3011$ & 201,5 & 0,517 \\
\hline
\end{tabular}

Таблица 3

\section{Показатели амплитуды ВКСП в группах исследуемых}

\begin{tabular}{|l|c|c|c|c|}
\hline \multirow{2}{*}{ Показатели } & Группа 1 & Группа 2 & U \\
\cline { 1 - 3 } & $\begin{array}{c}\text { Медиана; нижний квартиль; } \\
\text { верхний квартиль }\end{array}$ & $\begin{array}{c}\text { Медиана; нижний квартиль; } \\
\text { верхний квартиль }\end{array}$ & P \\
\hline A2 справа & 2,$1 ; 1,7 ; 2,2$ & 4,$3 ; 2,3 ; 4,8$ & 25,0 & 0,036 \\
\hline A1 справа & 0,$3 ; 0,1 ; 0,4$ & 1,$0 ; 0,1 ; 1,8$ & 23,5 & 0,030 \\
\hline
\end{tabular}

Выявляется достоверно более высокий уровень амплитуды компонента А1 и A2 в группе 2 исследуемых лиц.

Нами решалась задача определения связи между показателями вегетативного обеспечения деятельности и её результативностью в группах исследуемых при помощи логит-регрессионного анализа. В таблице 4 приведены характеристики данной модели.

Сходимость по критерию хи-квадрат составила 8,27 $\mathrm{p}=0,040$; оценки параметров статистически значимы. Корректное оценивание распределения исследуемых на группы с различной результативность осуществлено в 78\% случаев в группе 1 и 
Показатели модели логит-регрессии для прогнозирования результативности деятельности в группах

\begin{tabular}{|l|c|c|}
\hline \multicolumn{1}{|c|}{ Характеристики } & Коэффициенты регрессии & $\mathrm{p}$ \\
\hline Свободный член & 3,17 & 0,020 \\
\hline CКО ВCP & $-0,03$ & 0,040 \\
\hline VLF/HF & $-1,40$ & 0,040 \\
\hline Амплитуда A2 ВКСП слева & $-1,02$ & 0,010 \\
\hline
\end{tabular}

в $75 \%$ случаев в группе 2; отношение шансов для СКО 0,975, для амплитуды А2 ВКСП слева 0,358 , для отношения VLF/HF 0,246.

Кроме того, была создана ИНС, которая позволяла на основе комплекса физиологических показателей вегетативного обеспечения деятельности прогнозировать результативность деятельности практически здоровых лиц.

Данная ИНС по своей архитектуре являлась многослойным персептроном. ИНС включала 12 входных нейронов, 12 нейронов промежуточного слоя и 2 выходных нейронами (определяющими при их активации распределение испытуемых в группу 1 или 2), функция активации скрытых и выходным нейронов представляла собой экспоненту. Производительность обучения данной ИНС - $82 \%$, контрольная производительность - 75\%, тес-

товая производительность - 83\%.

В таблице 5 представлен ранжированный (в порядке убывания значимости для решения задачи классификации) список используемых ИНС показателей.

При оценке усреднённых рангов наибольшее значение имели статистические показатели ВСР (усреднённый ранг 5,3), второе место занимали показатели спектрального анализа ВСР (усреднённый ранг 6,4), третье место показатели ВКСП (усреднённый ранг 7,5).

На рисунке 1 представлена ROC кривая для ИНС.

При проведении кластерного анализа выявленная неоднородность групп исследуемых по результативности моделируемой деятельности позволяет определить группу 1 как низко результативную, а группу 2 как результативную.

Таблица 5

\section{Ранжированный список показателей вегетативного обеспечения деятельности, используемых ИНС для классификации исследуемых в группы с различной результативностью}

\begin{tabular}{|c|c|c|c|}
\hline $\begin{array}{c}\text { № } \\
\Pi / \Pi\end{array}$ & Показатели & $\begin{array}{c}\text { № } \\
\text { П/П }\end{array}$ & Показатели \\
\hline 1 & LF BCP & 7 & HF BCP \\
\hline 2 & TP BCP & 8 & ЧСС ВСР \\
\hline 3 & ИН ВСР & 9 & А А2 ВКСП справа \\
\hline 4 & А А1 ВКСП справа & 10 & VLF BCP \\
\hline 5 & СКО ВСР & 11 & А А2 ВКСП слева \\
\hline 6 & А А1 ВКСП слева & 12 & ИЦ ВСР \\
\hline
\end{tabular}

Примечание: А - амплитуда 


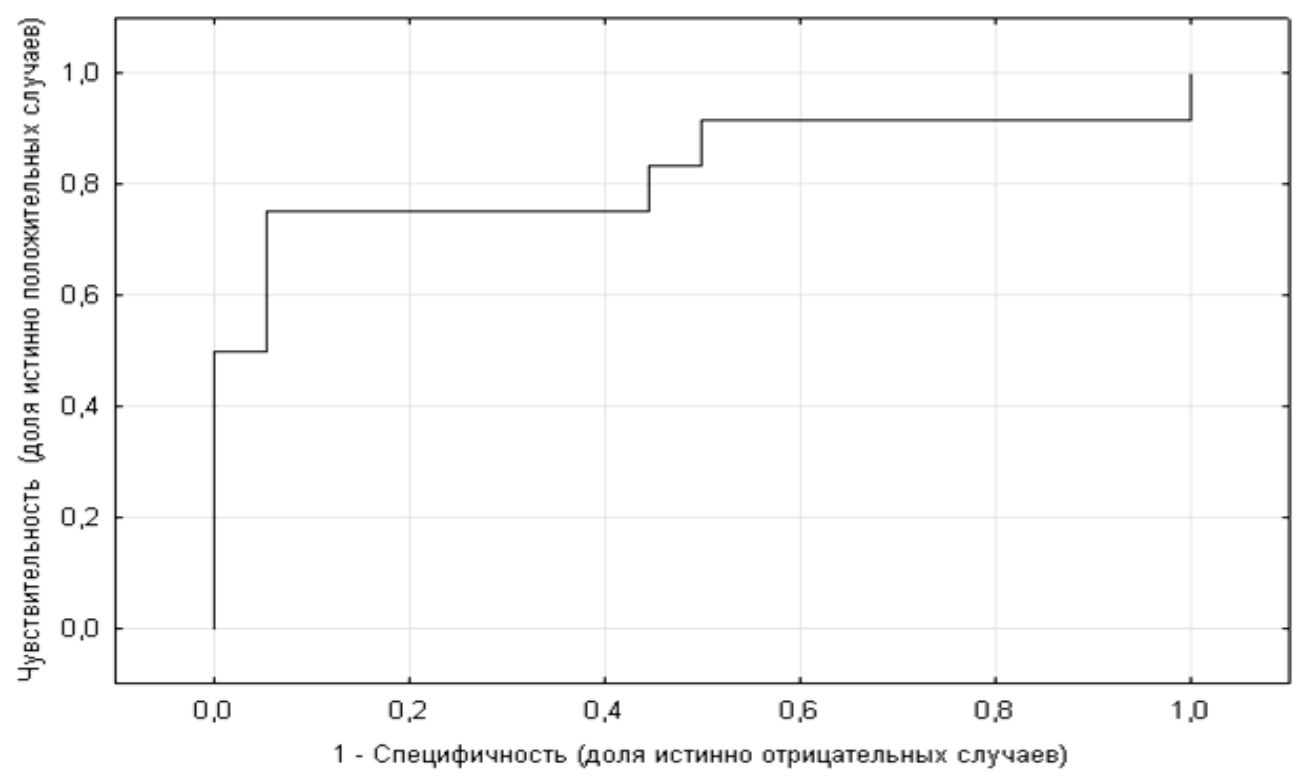

Рис. 1. ROC кривая для ИНС; площадь под кривой 0,824

При сравнительном анализе механизмов вегетативного обеспечения деятельности в группах достоверные различия выявлены по показателям ВКСП, отражающим увеличение как трофотропных (показатели амплитуды А1 ВКСП), так и эрготропных (показатели амплитуды А2 ВКСП) влияний в результативной группе исследуемых. Таким образом, определяется большая активация супрасегментарных механизмов вегетативной регуляции в результативной группе исследуемых, при этом эрготропные механизмы направлены на вегетативное обеспечение в период реализации деятельности, а трофотропные на восстановление физиологических ресурсов [8].

Модели логит-регрессионного анализа и технологии ИНС позволили достаточно эффективно разделять исследуемых на группы с различной результативностью поведения, что отражает роль механизмов вегетативного обеспечения в системной организации целенаправленной деятельности при формировании её неодинаковой результативности и соответствует литературным данным $[9,10]$.

Важно, что наибольшее значение в прогнозирование результативности деятельности как при использовании модели логит-регрессионного анализа, так и ИНС играют характеристики статистического анализа динамического ряда R-R интервалов, отражающие суммарный эффект влияния как сегментарных, так и супрасегментарных вегетативных субсистем [11]. Вместе с тем, технология ИНС позволяет за счёт расширения комплекса включённых в анализ параметров увеличить классификационную способность модели.

\section{Выводы}

1. Практически здоровые лица неоднородны по показателям моделируемой целенаправленной деятельности с выделением результативной и низко результативной групп.

2. Высокая результативность деятельности у практически здоровых лиц сопровождается большей активацией надсегментарных как эрготропных, так и трофотропных механизмов.

3. Технологии логит-регрессионного анализа и искусственных нейронных сетей позволяют осуществить прогнозирование результативности деятельности на основе физиологических показателей её вегетативного обеспечения.

Технология искусственных нейронных сетей обладает более высокой классификационной способностью в определении результативности деятельности за счёт увеличения количества анализируемых показателей вегетативного обеспе- 
чения целенаправленной деятельности.

\section{Дополнительная информация}

Конфликт интересов: отсутствует. Участие авторов:

Концепция и дизайн исследования - 3орин Р.А., Лапкин М.М., Жаднов В.А.
Сбор и обработка материала - Зорин Р.А., Медведева Ю.И., Курепина И.С.

Статистическая обработка данных - 3орин Р.А., Медведева Ю.И., Курепина И.С.

Написание текста - Зорин Р.А., Медведева

Ю.И., Лапкин М.М., Жаднов В.А.

Редактирование - Лапкин М.М., Жаднов В.А.

\section{Литература}

1. Вейн А.М., Голубев В.Л., ред. Вегетативные расстройства: Клиника, лечение, диагностика. М.: Медицинское информационное агентство; 2010.

2. Зорин Р.А., Лапкин М.М., Жаднов В.А. Моторно-вегетативные механизмы целенаправленной деятельности больных эпилепсией и клинические проявления заболевания // Российский медико-биологический вестник имени академика И.П. Павлова. 2017. Т. 25, №1. С. 76-85. doi: 10.23888/PAVLOVJ2017176-85

3. Sudakov K.V. Theory of functional systems: a keystone of integrative biology // Cognitive systems monographs. 2015. Vol. 25. P. 153-173. doi: 10.1007/978-3-319-19446-2_9

4. Похачевский А.Л, Рекша Ю.М., Гаджимурадов Ф.Р., и др. Алгоритмы, методы и аппаратура анализа временного ряда кардиоритмограммы при нагрузочном тестировании // Биомедицинская радиоэлектроника. 2018. №1. С. 33-38.

5. Котельников С.А., Ноздрачев А.Д., Одинак М.М., и др. Вызванные кожные вегетативные потенциалы (современные представления о механизмах) // Физиология человека. 2000. Т. 26, №5. C. 79-91.

6. Боровиков В.П. Популярное введение в современный анализ данных в системе Statistica. M.: Горячая линия - Телеком; 2013.

7. Moein S. Medical diagnosis using artificial neural networks. Hershey: Medical Information Science Reference; 2014. doi:10.4018/978-1-4666-61462.ch001

8. Меркулова М.А., Лапкин М.М., Зорин Р.А. Использование кластерного анализа и теории искусственных нейронных сетей для прогнозирования результативности целенаправленной деятельности человека // Наука молодых (Eruditio Juvenium). 2018. T. 6, №3. C. 374-382. doi:10.23888/HMJ201863374-382

9. Карлов В.А., Гнездицкий В.В., Деряга И.Н., и др. Эпилепсия и функциональная организация вегетативной нервной системы // Журнал неврологии и психиатрии им. С.С. Корсакова. 2013. Т. 113, №8. С. 4-9.

10. Рожнов О.И. Динамика параметров сердечнодыхательного синхронизма в сопоставлении с уровнем гормонов стресс-реализующей и стресс-лимитирующей систем при психогенном стрессе // Кубанский научный медицинский вестник. 2008. №6. С. 68-70.

11. Джебраилова Т.Д., Сулейманова Р.Г., Иванов Л.И., и др. Физиологическое обеспечение целенаправленной деятельности студентов во время компьютерного тестирования уровня знаний // Вестник новых медицинских технологий. 2013. T. 20, №1. C. 38-42.

\section{References}

1. Veyn AM, Golubev VL, editors. Vegetativnye rasstroystva: Klinika, lechenie, diagnostika. Moscow: Meditsinskoe informatsionnoe agentstvo; 2010. (In Russ).

2. Zorin RA, Lapkin MM, Zhadnov VA. The mechanisms of motor and vegetative maintenance of modeling activity in patients with epilepsy and clinical course of disease. I.P. Pavlov Russian Medical Biological Herald. 2017;25(1):76-85. (In Russ). doi:10.23888/PAVLOVJ2017176-85

3. Sudakov KV. Theory of functional systems: a keystone of integrative biology. Cognitive Systems Monographs. 2015;25:153-73. doi:10.1007/978-3319-19446-2_9

4. Pokhachevskiy AL, Reksha YuM, Gadzhimuradov FR, et al. Algorithms, methods and equipment for analysis of heart rate patterns in physical load testing. Biomedical Radioelectronics. 2018;(1):33-8. (In Russ).

5. Kotel'nikov SA, Nozdrachev AD, Odinak MM, et al. Vyzvannye kozhnye vegetativnye potentsialy (sovremennye predstavleniya o mekhanizmakh). Fiziologiya Cheloveka. 2000;26(5):79-91. (In Russ).

6. Borovikov VP. Populyarnoe vvedenie v sovremennyy analiz dannykh $v$ sisteme Statistica. Moscow: Goryachaya liniya - Telekom; 2013. (In Russ).

7. Moein S. Medical diagnosis using artificial neural networks. Hershey: Medical Information Science Reference; 2014. doi:10.4018/978-1-4666-61462.ch001

8. Merkulova MA, Lapkin MM, Zorin RA. The use of cluster analysis and the theory of artificial neural networks to predict the effectiveness of targeted human activity. Nauka molodykh (Eruditio Juvenium). 2018;6(3):374-82. doi:10.23888/HMJ2018 63374-382

9. Karlov VA, Gnezditskii VV, Depiaga IN, et al. Epilepsy and the functional organization of the autonomic nervous system. Zhurnal Nevrologii $i$ Psikhiatrii imeni S.S. Korsakova. 2013;113(8):4-9. 
(In Russ).

10. Pshennikova MG; Kryzhanovskii GN, editor. Stress: regulyatornye sistemy i ustoichivost' $k$ stressornym povrezhdeniyam. In: Dizregulyatsionnaya patologiya. Moscow: Meditsina; 2002. P. 307-24. (In Russ).
11. Dzhebrailova TD, Sulejmanova RG, Ivanova LI, et al. Physiological processes underlying purposeful activity in the students during computer testing. Journal of New Medical Technologies. 2013;20(1): 38-42. (In Russ).

\section{Информация об авторах [Authors Info]}

Зорин Роман Александрович - к.м.н., доцент кафедры неврологии и нейрохирургии, Рязанский государственный медицинский университет им. акад. И.П. Павлова, Рязань, Россия. e-mail: zorin.ra30091980@mail.ru

SPIN: 5210-5747, ORCID ID: 0000-0003-4310-8786.

Roman A. Zorin - MD, PhD, Associate Professor of the Department of Neurology and Neurosurgery, Ryazan State Medical University, Ryazan, Russian Federation. e-mail: zorin.ra30091980@mail.ru SPIN: 5210-5747, ORCID ID: 0000-0003-4310-8786.

Медведева Юлия Игоревна - очный аспирант кафедры неврологии и нейрохирургии, Рязанский государственный медицинский университет им. акад. И.П. Павлова, Рязань, Россия.

SPIN: 9025-8780, ORCID ID: 0000-0003-2292-6990.

Yuliya I. Medvedeva - PhD Student of the Department of Neurology and Neurosurgery, Ryazan State Medical University, Ryazan, Russian Federation.

SPIN: 9025-8780, ORCID ID: 0000-0003-2292-6990.

Курепина Инна Сергеевна - очный аспирант кафедры неврологии и нейрохирургии, Рязанский государственный медицинский университет им. акад. И.П. Павлова, Рязань, Россия.

SPIN: 6914-4106, ORCID ID: 0000-0001-9207-2447.

Inna S. Kurepina - PhD Student of the Department of Neurology and Neurosurgery, Ryazan State Medical University, Ryazan, Russian Federation.

SPIN: 6914-4106, ORCID ID: 0000-0001-9207-2447.

Лапкин Михаил Михайлович - д.м.н., профессор, зав. кафедрой нормальной физиологии с курсом психофизиологии, Рязанский государственный медицинский университет им. акад. И.П. Павлова, Рязань, Россия.

SPIN: 5744-5369, ORCID ID: 0000-0003-1826-8307.

Michael M. Lapkin - MD, PhD, Professor, Head of the Department of Physiology with the Psychophysiology Course, Ryazan State Medical University, Ryazan, Russian Federation.

SPIN: 5744-5369, ORCID ID: 0000-0003-1826-8307

Жаднов Владимир Алексеевич - д.м.н., профессор, зав. кафедрой неврологии и нейрохирургии, Рязанский государственный медицинский университет им. акад. И.П. Павлова, Рязань, Россия.

SPIN: 1632-5083, ORCID ID: 00000-0002-5973-1196.

Vladimir A. Zhadnov - MD, PhD, Professor, Head of the Department of Neurology and Neurosurgery, Ryazan State Medical University, Ryazan, Russian Federation.

SPIN: 1632-5083, ORCID ID: 00000-0002-5973-1196.

Цитировать: Зорин Р.А., Медведева Ю.И., Курепина И.С., Лапкин М.М., Жаднов В.А. Вегетативное обеспечение целенаправленной деятельности и её результативность у практически здоровых лиц // Наука молодых (Eruditio Juvenium). 2019. T. 7, №1. С. 38-45. doi:10.23888/HMJ20197138-45

To cite this article: Zorin RA, Medvedeva YuI, Kurepina IS, Lapkin MM, Zhadnov VA. The vegetative support of purposeful activity and perfomance efficiency in healthy people. Science of the young (Eruditio Juvenium). 2019;7(1):38-45. doi:10.23888/HMJ20197138-45

Поступила / Received: 01.04.2018

Принята в печать / Accepted: 20.03.2019 